\title{
Demonstration of a microgrid manager in a distribution grid with high PV penetration and Storage: La Graciosa case study
}

\author{
Guillermo Domínguez-López ${ }^{1, *}$, Pol Paradell-Solà ${ }^{1, *}$, José Luis Domínguez-García ${ }^{1, *}$, \\ Jacob Rodríguez-Rivero ${ }^{2}$, and Jorge Sánchez-Cifuentes ${ }^{2}$ \\ ${ }^{1}$ Catalonia Institute for Energy Research (IREC), 08930 Sant Adrià de Besòs, Spain \\ ${ }^{2}$ Endesa Distribución, Spain
}

\begin{abstract}
Future power systems with high penetration of distributed energy resources (DER) and information and communication technology (ICT) will allow increasing the controllability and observability of the grid. However, new challenges for low voltage distribution networks has arisen where the increasing DER is mainly photovoltaic (PV) installed on the roofs of buildings. This type of generation is variable and generates disturbances in the network. Islands are ideal to experience and install new technologies since are weak grids and the penetration of PV will lead to bidirectional power flows at distribution level, thus a smart control will be required. This article aims to present and innovative tool for the distribution system operators (DSOs) to monitor and manage grid operation. In near future, both DER and client's flexibility will increase. New monitoring and control tools for DSOs are essential. Furthermore, the grid operation optimization is needed to accommodate the expected DER installations and the active participation of consumers' flexibility services in markets ensuring system efficiency. This tool is tested on "La Graciosa" grid, one of the Canary Islands, to show its benefit. The grid studied includes a hybrid energy storage system (HESS), PV generators, controllable/uncontrollable loads and equipment to monitor power flows.
\end{abstract}

\section{Introduction}

Distributed energy resources (DER) are expected to take the lead of future power systems which have to be prepared for the increasing penetration of distributed generation (DG) at distribution level of the grid. A group of DER and loads in the distribution system is a microgrid (MG) [1-5]. A lot of methods and power management strategies are being developed [5-10] including DG, storage, controllable loads and other elements. However, these studies are focused on develop optimization methods for MG in simulation. There is a need for new monitoring and control tools for distribution system operators (DSOs) and land these tools from concepts to real world.

\footnotetext{
*Corresponding authors : gdominguez@irec.cat, pparadell@irec.cat, jldominguez@irec.cat
} 
This paper presents a microgrid manager (MGM) tested in a pilot experiment done on "la Graciosa" island, which is located in the Canary Islands. It is worth noting that "La Graciosa" is the perfect network to validate and demonstrate the advantages of microgrids. The MGM consists on a microgrid optimizer and a supervisory control and data acquisition (SCADA) application. The performance and benefits of the MGM deployed are mentioned and also a brief explanation of lessons learned from the pilot that need to be considered when moving from simulations to real.

Part of this study has been carried out and validated on Project "GRACIOSA" which main partners are "Endesa Distribución", "Endesa Energía", "Cuadros Eléctricos Nazarenos" (CEN) and "Instituto Tecnológico de Canarias" (ITC) together with associate partners as IREC, Comillas, FIDETIA, ULL and AICIA.

\section{Microgrid optimizer}

The microgrid optimizer manages all the flexible assets existing on the distribution grid to make the network more reliable. It runs each 15 minutes and makes an optimization of 24 hours in steps of 15 minutes. A scheme of how data is flowing from the sensors to the set points send is shown in Fig 1. The devices considered in the system are explained in the next section with the SCADA and test scenario.

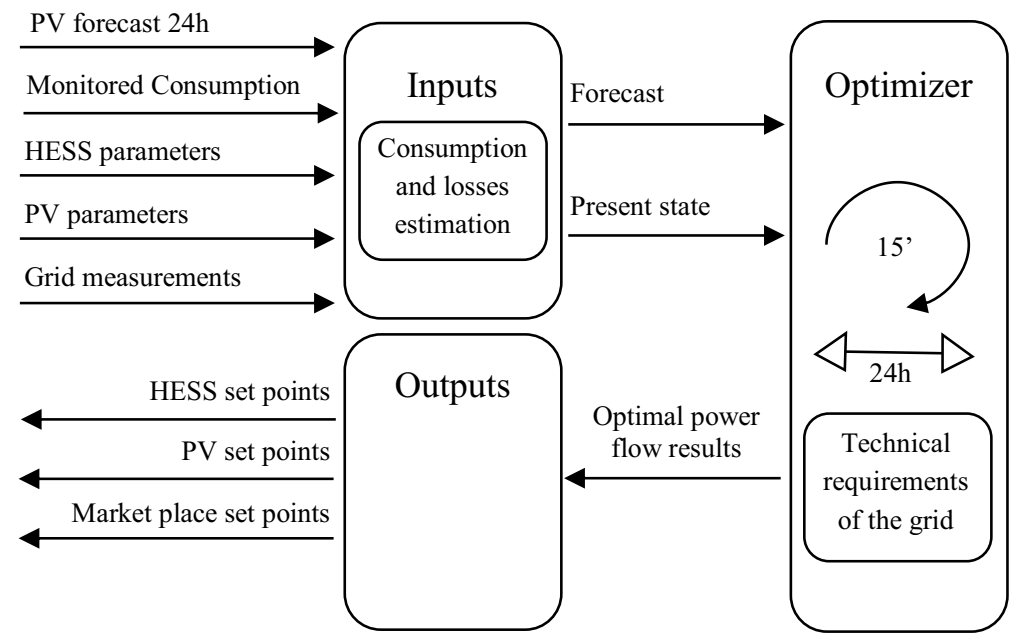

Fig. 1. Optimization method from sensor to set point.

The sensors provide data from the devices connected to the microgrid and grid measurements done along the system. Also, a PV forecast is used to estimate the consumption and losses of the microgrid. With historical data, a forecast for the consumption is also done and these inputs are served to the optimizer to run the optimal power flow. The results for the 24 hour prediction optimal power flow of the microgrid are gathered to send the appropriate set points to the devices at the correct time.

The optimization uses a three-phase balanced AC model of power flow with polar variables. It solves a multi-objective optimization maximizing the PV generation and minimizing the power losses of the distribution grid. The equations can be seen at:

$$
\begin{gathered}
\max f(P G E N)=\sum_{t \in[1, n t]}\left(\sum_{i \in[1, n P V]} P V_{i, t}-\sum_{(n, k) \in L \cup(i, i)} \text { Plos }_{n, k, t}\right) \\
\text { Ploss }_{n, k, t}=G_{n, k}\left(V_{n} \sin \delta_{n, t}-V_{k} \sin \delta_{n, t}\right)^{2}+B_{n, k}\left(V_{n} \sin \delta_{n, t}-V_{k} \sin \delta_{n, t}\right)^{2}
\end{gathered}
$$


Also to accomplish the purpose of the microgrid optimizer, the model has physical constraints as voltage limits of the network, power limits of each branch and the state of charge limits for the batteries.

The language used to manage this program is Julia with some complements. Julia is a high-level dynamic programming language designed to address the needs of highperformance numerical analysis and computational science. Also, the program uses the Bonmin solver, installed on Julia, to resolve the non-lineal equations of the model.

\section{SCADA and test scenario}

In this section, the test scenario and the SCADA are presented. Two lines with high PV penetration of the whole distribution grid of the "La Graciosa" are in the scope of the pilot and they can be seen together with the equipment installed in the grid in Fig. 2 which is one of the screens of the SCADA developed in an industrial tool (Wonderware by Schneider Electric). The system consists of 6 power analysers, a Hybrid Energy Storage System (HESS), 2 PV installations, 2 cameras for PV forecast and, finally, 3 self-consumption (PV with storage) installations have been deployed in clients to provide flexibility.

All devices have been integrated in the SCADA so the application can send set points from the optimizer and store data in real time, as it can be seen in Fig. 3. The communications from the SCADA server to the equipment deployed is based on Modbus TCP and connected by $3 \mathrm{G}$ to internet.

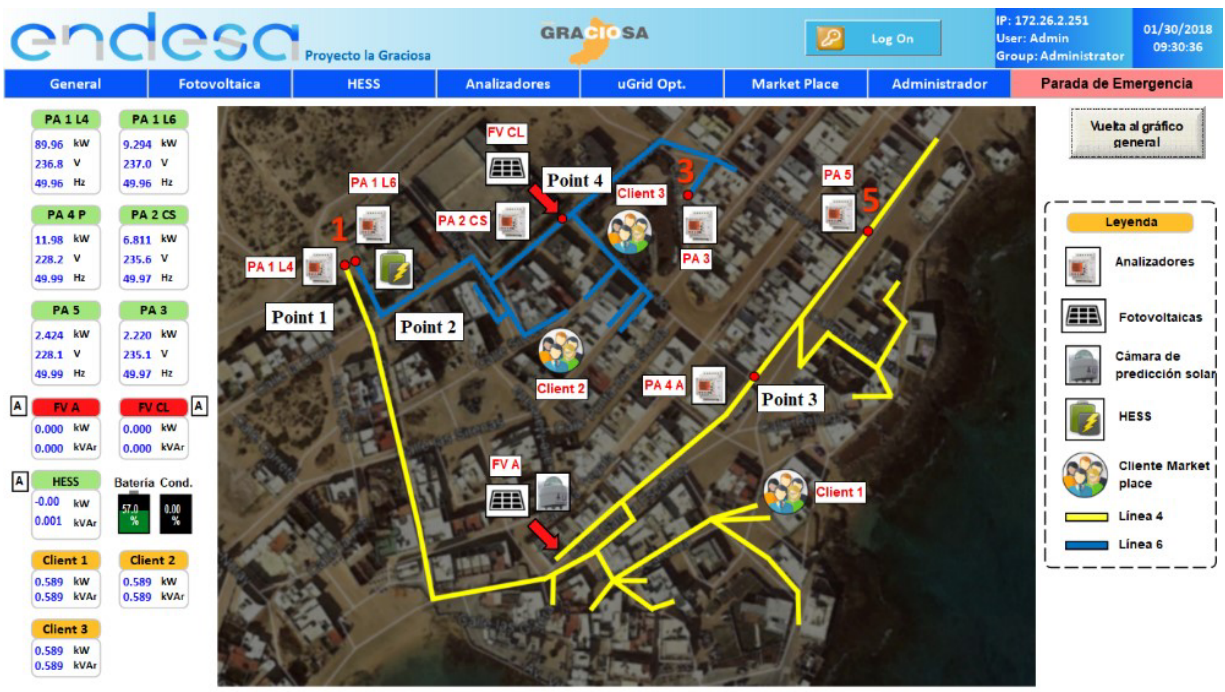

Fig. 2. Lines involved and equipment installed in "la Graciosa" island. 


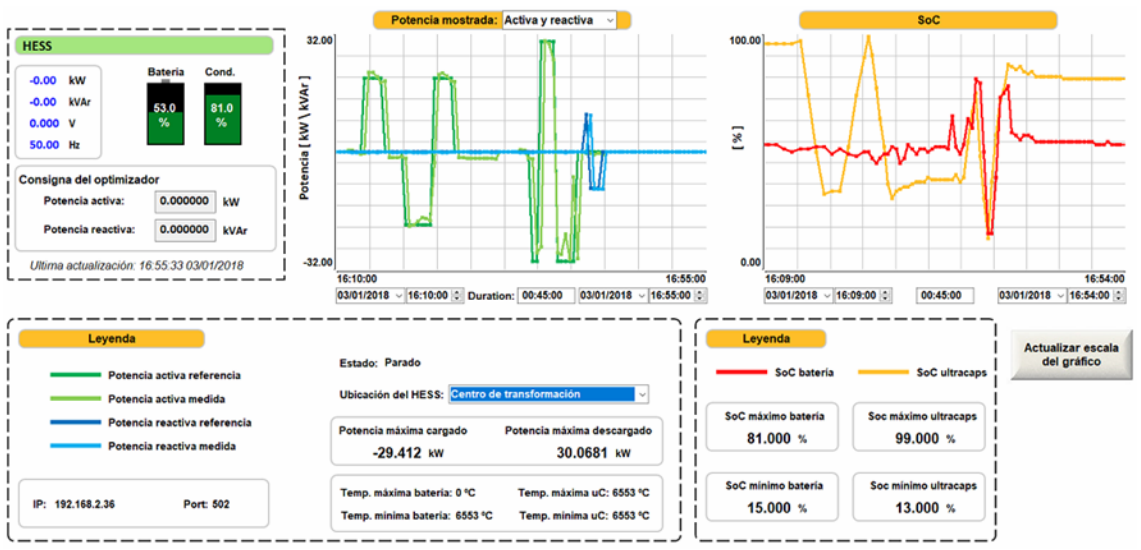

Fig. 3. Real-time monitoring of HESS performance during the test.

\section{Tool capabilities and main test results}

The equipment installed and the tool deployed increase the grid functionalities and upgrade the system into a smart microgrid. The SCADA enables to manage the system locally and remotely while it gathers information from the equipment in the grid to real-time monitor it. Therefore, the DSO is able to detect grid events at distribution level and study data stored to enhance power supply security and reliability. To illustrate this point, a discharge of the grid during the test is shown in Fig. 4 where the voltage and the power flowing in the line droop to zero and autonomously the tool reconnects all the equipment when the power supply is restored.

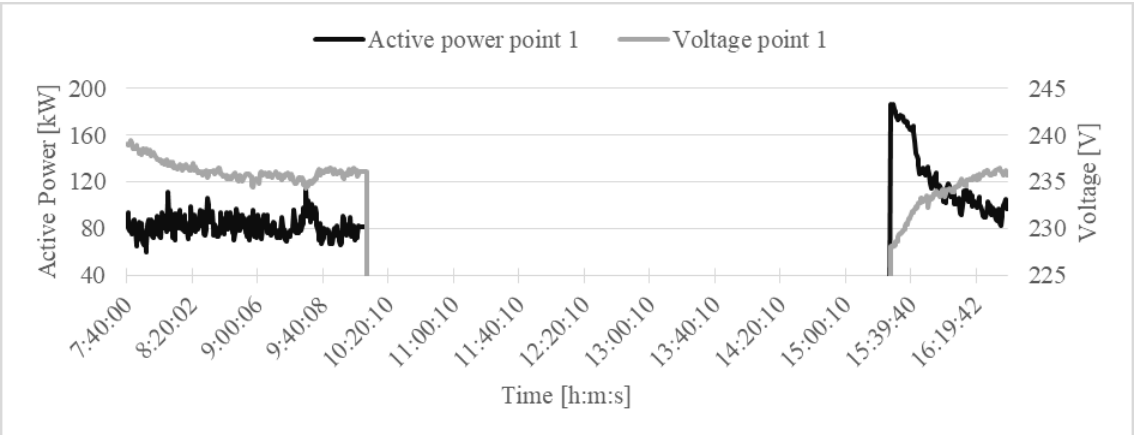

Fig. 4. Real-time monitoring of a discharge and reconnection during the test.

Moreover, the SCADA offers the possibility of remotely operate DER and HESS to change their low level control strategy and turn on, turn off or reset the device if a malfunction appears during real-time management. This new asset avoids to send a technical operator by the DSO for minor operations and offers an increase of the system controllability.

Moving to the main test results of the optimizer, the first indicator that is calculated is the accuracy of the optimizer prediction. The accuracy is defined as equal to the error between the prediction expected by the optimizer and the real power measured by the power analysers. These errors calculated at some measurement points provide a general overview of the accuracy of the PV and demand forecasts and, in fact, the performance of the optimizer and set points sent to the smartgrid active actors. 
As usual when simulation analysis reach a pilot, the test came across real world unexpected problems which had to be solved as they showed up. The main problems faced during the test were communication problems between the SCADA server and the rest of the equipment due to weak $3 \mathrm{G}$ internet connection in the island. These problems had interaction with the PV and demand calculation, but as the accuracy is intended to be calculated considering normal operation with no communication problems, the affected values by these problems are underestimated. The accuracy is calculated for 4 points of measure that can be seen in Fig. 2. The function which calculates the accuracy is defined by equation 3 and its values are presented in Table 1.

$$
\operatorname{Error}(t)=[\text { Value_measured }(t)-\text { Value_prediction }(t)] /(\text { Nominal Line Power })^{\dagger}
$$

Table 1. Maximum and average error calculated for the most relevant measurement points.

\begin{tabular}{|c|c|c|c|c|}
\hline & Point 1 (Line 4) & Point 2 (Line 6) & Point 3 (Line 4) & Point 4 (Line 6) \\
\hline Maximum \% Error & $\pm 22 \%$ & $\pm 16 \%$ & $\pm 9 \%$ & $\pm 10 \%$ \\
\hline Average \% Error & $7 \%$ & $4 \%$ & $2 \%$ & $4 \%$ \\
\hline
\end{tabular}

The average error is lower than $7 \%$ for all the measurement points, so the accuracy of the microgrid optimizer is high given the number of measurement points for the two lines and the uncertainty of the system. The power analysers have an own confidence interval in its measurements, usually $\pm 0,5 \%$, so the average error of the optimizer is potentially lower if this tolerance is removed. Furthermore, it can be seen in Fig. 5 how the error is mainly greater during the PV production hours and the maximum error found from the whole demonstrator is $\pm 22 \%$. This fact is related with several variables that can influence, but it is mainly a sign of the accuracy of the demand and PV forecast which is highly erratic.

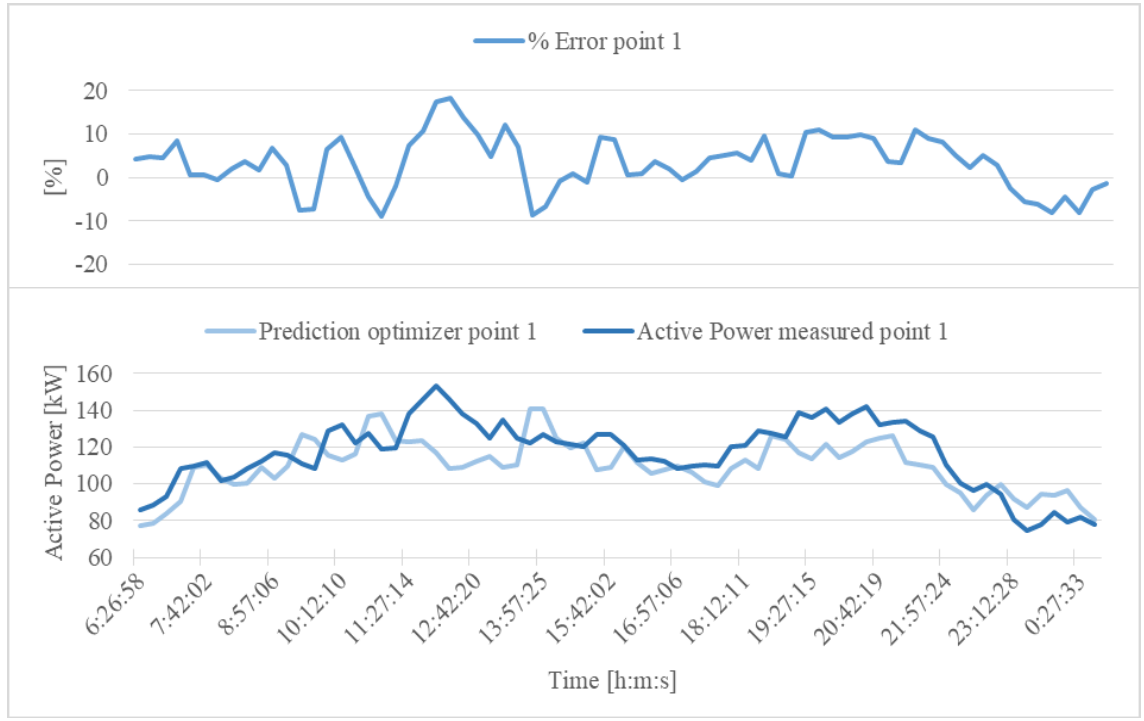

Fig. 5. Active power prediction, real value and error in time domain for point 1.

\footnotetext{
${ }^{\dagger}$ Nominal Line Power: Nominal power value of each line depending on point of measure.

$¥$ Average error: Calculated through the root mean square error.
} 
In addition, another study is done to check if the clients of the Market Place are able to follow the set points calculated by the microgrid optimizer. The algorithm calculates the maximum power consumption of the clients to reach the technical requirements of the grid so, theoretically, the Clients should not exceed this consumption. The consumption and the maximum power consumption expected of two Clients are shown in Fig. 6.

The three Clients follow the maximum power consumption requirement from $87 \%$ to $91 \%$ of the test time. Thus, the consumption expected is exceeded between $9 \%$ and $13 \%$ of the total time and this fact affects on the microgrid optimizer accuracy. In addition, the maximum error between the measured consumption and the maximum expected consumption calculated by the optimizer is presented in Table 2 . The function to calculate the error is given by equation 4 .

$$
\operatorname{Error}(t)=[\text { Cons_meas }(t)-\text { Max_cons_predic }(t)] /(\text { Contracted Power }) \S
$$

Table 2. Maximum error calculated for the clients of the market place.

\begin{tabular}{|c|c|c|c|}
\hline & Client 1 & Client 2 & Client 3 \\
\hline Maximum \% Error & $1,8 \%$ & $69 \%$ & $54 \%$ \\
\hline
\end{tabular}

This error can be up to $69 \%$, so it is clearly a source of deviations in the microgrid optimizer and, as a consequence, the set points sent to the PV inverters and to the HESS do not reach the optimum point of operation of the system.

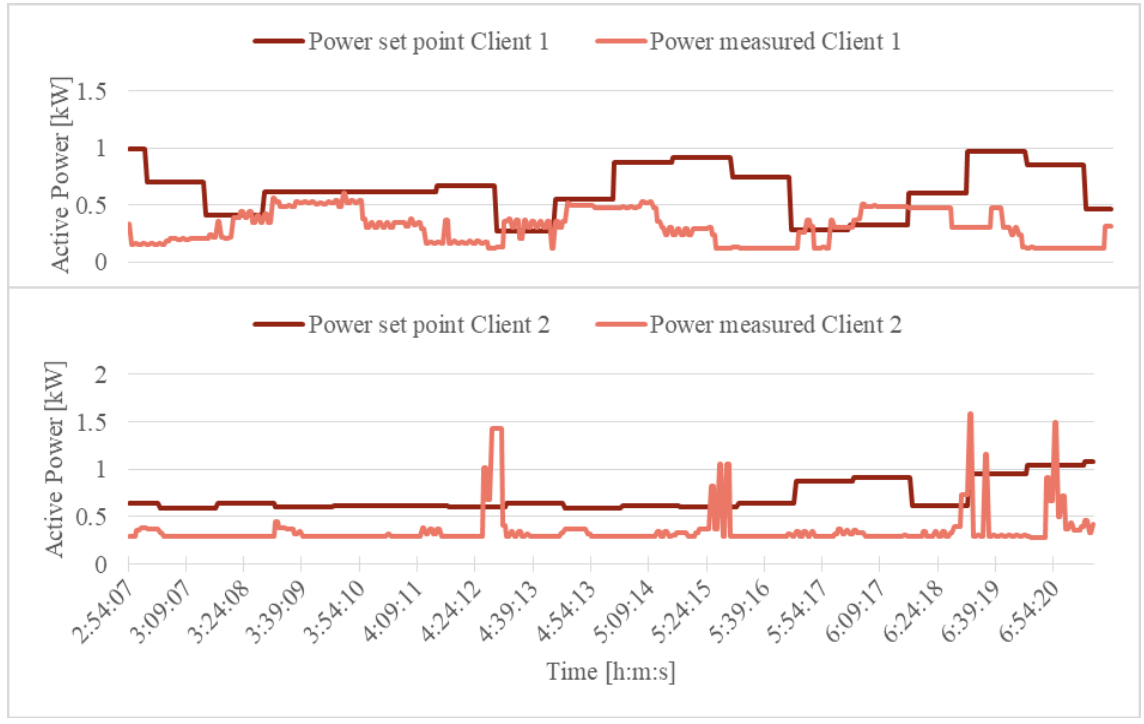

Fig. 6. Power consumption and maximum consumption expected for clients 1 and 2 in time domain.

\section{Conclusion and next steps}

This paper proposed an optimization strategy for microgrids with DER and HESS together with a centralized communication structure based on a SCADA application. Both together, optimization and SCADA, have been tested in a real pilot on "la Graciosa" island. Finally, a SCADA is found to be a great implementation to upgrade conventional distribution grids into

$\S$ Contracted Power: Considered as each Client Contracted Power. 
smartgrids. The accuracy of the predictions of the optimizer and its performance is acceptable, but highly dependent on DG and demand forecast accuracy. Real-time low level controls are needed to compensate the error given by a non-accurate forecast or communication problems during normal operation. In a future local market where clients will be benefited for offering flexibility to the system, they should be penalized when they do not accomplish the requirements.

There are mainly three actions that could potentially upgrade the automatization process and better performance of the optimizer. A first action would be to upgrade the communications structure switching $3 \mathrm{G}$ connection into a wifi to connect all the devices together Also, a suitable protocol for internet of things such as LoRa would increase the reliability of the remote connection and quality of the data. The optimization is highly dependent on the forecast for both solar and consumption, so a second action would be to improve the knowledge of the distribution grid, the forecasting and deployment of more points of measurement along the lines. Finally, a real time module could be implemented to balance the power set points calculated to adjust the forecast with the real powers flowing in the microgrid.

Future work is focused on improve the system with three phase imbalance conditions as the low voltage microgrid studied has several loads single-phase connected. Currently the system is studied under normal conditions of the microgrid, but the microgrid needs to be improved and test under emergency state. Also, a next step would be integrate more renewable generation and test the system islanded from the main grid. Nevertheless, an implementation of a strategy like Machine Learning to boost the system forecast for PV and consumption would be a next step into grid state estimation minimizing the power analysers deployed.

\section{Acknowledgments}

This research has been carried out by Project "GRACIOSA" funded by the programme INTERCONNECTA (FEDER-CDTI funds).

\section{References}

1. N. D. Hatziargyriou and A. P. S. Meliopoulos, "Distributed energy sources: Technical challenges". IEEE Power Eng. Soc. Winter Meeting, 2, 1017-1022, (2002).

2. R. H. Lasseter and P. Piagi, "Microgrid: A conceptual solution”. IEEE Power Electron., 6, 4285-4290, (2004).

3. H. Jiyaji, J. Chuanwen and X. Rong, "A review on distributed energy resources and MicroGrid". Renew. Sust. Energ. Rev., 12, 2472-2483, (2007).

4. S. M. Nosratabadi, R. Hooshmand, and E. Gholipour, "A comprehensive review on microgrid and virtual power plant concepts employed for distributed energy resources scheduling in power systems". Renew. Sust. Energ. Rev., 67, 341-363, (2017).

5. F. Katiraei and M.R. Iravani, "Power Management Strategies for a Microgrid with Multiple Distributed Generation Units". IEEE T. Power Syst., 21, 1821-1831, (2016).

6. A. Maulik and D. Das, "Power Flow and Economic Dispatch of a Microgrid in Presence of Feeder Flow Control Distributed Generators". $20176^{\text {th }}$ International Conference on Computer Applications In Electrical Engineering-Recent Advances (CERA), 187-192, (2017).

7. J. Qiu, J. Zhao, Y. Zheng, Z. Dong, Z. Y. Dong, "Optimal allocation of BESS and MT in a microgrid”. IET Gener. Transm. Dis., 12, 1988-1997, (2018). 
8. A. Maulik and D. Das, "Optimal operation of microgrid using four different optimization techniques”. Sustain. Energy Techn. Assess., 21, 100-120, (2017).

9. M. Lei, Z. Yang, Y. Wang, H. Xu, L. Meng, J. C. Vasquez and J. M. Guerrero, "Design of Energy Storage Control Strategy to Improve the PV System Power Quality". IEEE Ind. Elec., 2022-2027, (2016).

10. Y. Zhu, F. Zhuo, H. Shi, "Power Management Strategy Research for a PhotovoltaicHybrid Energy Storage System”. 2013 IEEE ECCE Asia Downunder, 842-848, (2013). 